\title{
Many-Body Dephasing in a Trapped-Ion Quantum Simulator
}

\author{
Harvey B. Kaplan $\odot,{ }^{1, *}$ Lingzhen Guo $\odot,{ }^{2,},{ }^{*} \dagger$ Wen Lin Tan, ${ }^{1}$ Arinjoy De $\odot,{ }^{1}$ Florian Marquardt, ${ }^{2,3}$ \\ Guido Pagano $\oplus^{1,4}$ and Christopher Monroe ${ }^{1, \$}$ \\ ${ }^{1}$ Joint Quantum Institute, Department of Physics and Joint Center for Quantum Information and Computer Science, \\ University of Maryland, College Park, Maryland 20742, USA \\ ${ }^{2}$ Max Planck Institute for the Science of Light, Staudtstrasse 2, 91058 Erlangen, Germany \\ ${ }^{3}$ Physics Department, University of Erlangen-Nuremberg, Staudtstrasse 5, 91058 Erlangen, Germany \\ ${ }^{4}$ Department of Physics and Astronomy, Rice University, 6100 Main Street, Houston, Texas 77005, USA
}

(Received 4 February 2020; accepted 20 August 2020; published 18 September 2020)

\begin{abstract}
How a closed interacting quantum many-body system relaxes and dephases as a function of time is a fundamental question in thermodynamic and statistical physics. In this Letter, we analyze and observe the persistent temporal fluctuations after a quantum quench of a tunable long-range interacting transverse-field Ising Hamiltonian realized with a trapped-ion quantum simulator. We measure the temporal fluctuations in the average magnetization of a finite-size system of spin-1/2 particles. We experiment in a regime where the properties of the system are closely related to the integrable Hamiltonian with global spin-spin coupling, which enables analytical predictions for the long-time nonintegrable dynamics. The analytical expression for the temporal fluctuations predicts the exponential suppression of temporal fluctuations with increasing system size. Our measurement data is consistent with our theory predicting the regime of manybody dephasing.
\end{abstract}

DOI: 10.1103/PhysRevLett.125.120605

Introduction.-Investigating the relaxation and dephasing dynamics of a closed many-body quantum system is of paramount importance to the study of thermodynamics and statistical physics. Most commonly, this problem is investigated by studying the time evolution of the expectation value of a local observable, e.g., particle density or magnetization, after quenching the system from an initial out-of-equilibrium state [1-4]. For a generic non(near-) integrable system, the expectation value tends to relax to a constant in the thermodynamic limit which can be described by a (pre)thermal state at some temperature depending on the initial state [5-22]. However, if the system size is finite, there exist persistent temporal fluctuations around the constant average value, as sketched in Fig. 1(a). Importantly, these persistent temporal fluctuations in the expectation value after a quench are distinct from the usual fluctuations of observables in equilibrium (where expectation values are constant). Studying these temporal fluctuations represents the next level of the description of quench dynamics going beyond merely looking at long-time observable averages.

Published by the American Physical Society under the terms of the Creative Commons Attribution 4.0 International license. Further distribution of this work must maintain attribution to the author(s) and the published article's title, journal citation, and DOI. Open access publication funded by the Max Planck Society.
A crucial question for statistical physics is how the temporal fluctuations are suppressed with increasing system size $N$. In the case of integrable systems mappable

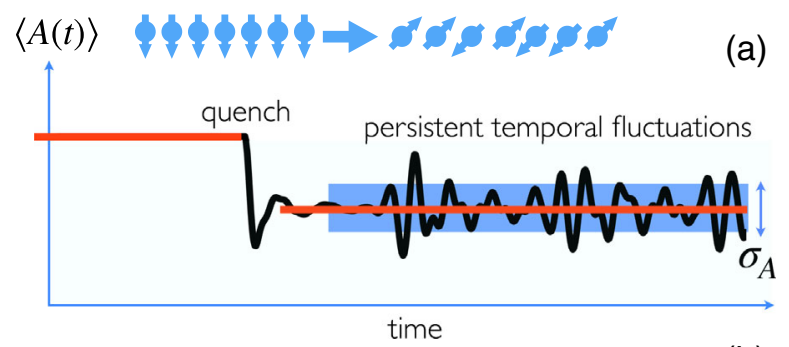

(b)
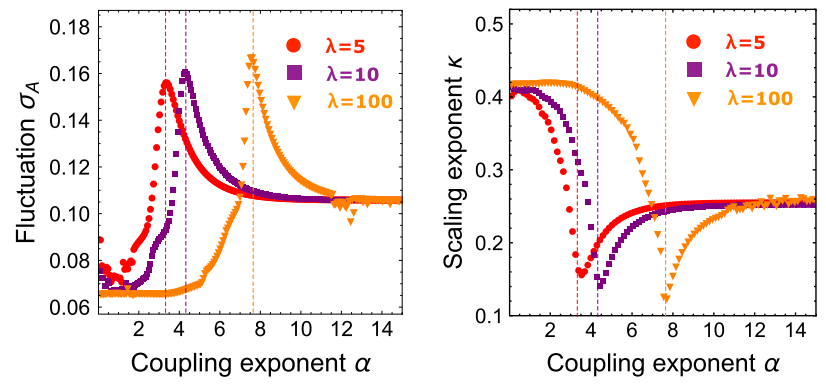

FIG. 1. (a) Schematic behaviour of an observable $\langle A(t)\rangle$ after a quench, in a finite-size system. (b) Temporal fluctuation $\sigma_{A}$ for $N=7$ spins (left) and size scaling exponent $\kappa$ (right) as a function of power-law coupling exponent $\alpha$ for three fixed parameters $\lambda=2 J_{0} / B$. The vertical dashed lines indicate the crossover values of $\alpha^{*}=\ln (2|\lambda|) / \ln 2$ [23]. 
to free quasiparticles, it has been found that the variance of temporal fluctuations scales as $1 / N$ [32-34]. In the case of generic nonintergrable systems [35-39], or the integrable systems solvable with the Bethe ansatz (not mappable to noninteracting systems) [40], the temporal fluctuations are exponentially suppressed by the system size due to the highly nondegenerate spectrum. This was first found only numerically. However, in Ref. [39], the authors were able, for the first time, to provide an exact analytical result for the exponential scaling of fluctuations with $N$ spins in a weakly nonintegrable system. In this setting, they identified a general dynamical regime which they termed "manybody dephasing" [41]. In the thermalization process, the dephasing mechanism comes from the relaxation of the quasiparticle distribution to thermal equilibrium by quasiparticle scattering described by the Boltzmann equation. In contrast, many-body dephasing results from lifting of all the exponentially large degeneracies of transition energies in integrable systems while the quasiparticle distribution can remain practically unchanged [39].

Nevertheless, the exponential size scaling due to manybody dephasing in nonintegrable systems has not yet been verified in experiments. Here, we give the first experimental observation of persistent temporal fluctuations after a quantum quench characterized as a function of system size, employing a trapped-ion quantum simulator. We present a direct measurement of relaxation dynamics in the nonintegrable system by measuring the temporal fluctuations in the average magnetization of a finite-size system of spin- $1 / 2$ particles. After including the experimental noise in the data analysis, the temporal fluctuations from experimental data are consistent with our numerical simulations and theoretical analysis based on the concept of many-body dephasing.

Model Hamiltonian.-The Hamiltonian implemented in this experiment is the long-range transverse-field Ising model,

$$
H=\sum_{i<j} J_{i j} \sigma_{i}^{x} \sigma_{j}^{x}-\frac{1}{2} B \sum_{i} \sigma_{i}^{z}
$$

where $J_{i j} \approx J_{0} /|i-j|^{\alpha}>0$, is a long-range coupling that falls off approximately as a tunable power law. The Hamiltonian (1) is implemented using an applied laser field which creates spin-spin interactions through spindependent optical dipole forces [42]. The spin chain is initialized to the $|\downarrow \downarrow \cdots \downarrow\rangle_{z}$ state, then a quench is performed using Hamiltonian (1), and the magnetization along the $z$ axis is measured as a function of time. The cases of $\alpha^{-1}=0$ and $\alpha=0$ correspond to two integrable limits, i.e., the nearest neighbor coupling and global coupling models, respectively. For a finite $\alpha>0$, Hamiltonian (1) is in general nonintegrable.

Temporal fluctuations. - In the present experiment, the observable is the magnetization, i.e., $A=N^{-1} \sum_{j} \sigma_{j}^{z}$. The temporal average of the variable $\langle A(t)\rangle$ is calculated as $\overline{\langle A(t)\rangle} \equiv T^{-1} \int_{t_{i}}^{t_{i}+T}\langle A(t)\rangle d t$, where the temporal averaging is restricted within the time window between $t_{i}$ and $t_{i}+T$. The variance of temporal fluctuations of $\langle A(t)\rangle$ is defined via $\sigma_{A}^{2} \equiv \overline{[\langle A(t)\rangle-\overline{\langle A(t)\rangle}]^{2}}$, with $\sigma_{A}$ the standard deviation. We use $\left|\Phi_{n}\right\rangle\left(n=1,2 \cdots 2^{N}\right)$ to represent the manybody eigenstates of Hamiltonian (1) with eigenenergy $E_{n}$. Given the initial state $|\psi(0)\rangle$, the exact time evolution of the observable is $\langle A(t)\rangle=\sum_{m, n}\left\langle\psi(0) \mid \Phi_{m}\right\rangle$ $\left\langle\Phi_{m}|A| \Phi_{n}\right\rangle\left\langle\Phi_{n} \mid \psi(0)\right\rangle e^{i \Delta_{m n} t}$, where $\Delta_{m n} \equiv E_{m}-E_{n}$ is the transition energy between the two energy levels $\left|\Phi_{m}\right\rangle$ and $\left|\Phi_{n}\right\rangle(\hbar=1)$. In the long time window limit $(T \rightarrow+\infty)$, we have the average,

$$
\overline{\langle A(t)\rangle}=\sum_{m, n, \Delta_{m n}=0}\left\langle\psi(0) \mid \Phi_{m}\right\rangle\left\langle\Phi_{m}|A| \Phi_{n}\right\rangle\left\langle\Phi_{n} \mid \psi(0)\right\rangle,
$$

and the variance of temporal fluctuation,

$\sigma_{A}^{2}=\sum_{\Delta \neq 0}\left|\sum_{\Delta_{m n}=\Delta}\left\langle\psi(0) \mid \Phi_{m}\right\rangle\left\langle\Phi_{m}|A| \Phi_{n}\right\rangle\left\langle\Phi_{n} \mid \psi(0)\right\rangle\right|^{2}$,

with $\Delta$ denoting the set of all the possible values of $\Delta_{m n}$. For the integrable models $\left(\alpha=0\right.$ or $\left.\alpha^{-1}=0\right)$, there are exponentially many degeneracies with the number of spins for a given transition energy $\Delta_{m n}$, since each many-body eigenstate can be labeled by many independent conserved quantities. However, for a generic nonintegrable model $(\alpha>0)$, there are no conserved quantities except the Hamiltonian itself. Thus, it is reasonable to assume that all the degeneracies of transition energies are lifted, making $\Delta_{m n}=0$ only possible for $m=n$ in the nonintegrable model, so Eq. (2) simplifies to

$$
\sigma_{A}^{2}=\sum_{m \neq n}\left|\left\langle\psi(0) \mid \Phi_{m}\right\rangle\left\langle\Phi_{m}|A| \Phi_{n}\right\rangle\left\langle\Phi_{n} \mid \psi(0)\right\rangle\right|^{2} .
$$

Upon closer analysis, this is the basic reasoning that leads to the exponential suppression of fluctuations with system size [35]. However, in general cases, it is impossible to evaluate this expression analytically.

Theoretical results.-We investigate numerically the temporal fluctuation $\sigma_{A}$ as a function of $\alpha$ for fixed dimensionless parameter $\lambda \equiv 2 J_{0} / B$. We also extract from our numerical simulations the size scaling exponent $\kappa$ from the fit $\sigma_{A} \propto e^{-\kappa N}$ for $N=3-10$ spins [see Fig. 1(b)]. We find two distinct regimes, at small and large $\alpha$, separated by the crossover value of $\alpha^{*}=\ln (2|\lambda|) / \ln 2$ [23]. The crossover between those regimes can be understood from the competition between the two terms in Hamiltonian (1), i.e., the magnetic field energy $-B \sum_{i} s_{i}^{z}$ (where $s_{i}^{z} \equiv \frac{1}{2} \sigma_{i}^{z}$ ) and the next-nearest-neighbor (NNN) spin-spin coupling $2^{-\alpha+2} J_{0} \sum_{i} s_{i}^{x} s_{i+2}^{x}$, which, for $\alpha>0$, is the leading term responsible for breaking integrability [23]. In the regime of $\alpha \gg \alpha^{*}$, by neglecting the NNN (and other long-range) 
coupling terms, the Hamiltonian is reduced into an integrable model. Adding the NNN coupling terms weakly breaks the integrability and results in many-body dephasing [39]. We cannot reach this regime in the experiment since the power-law exponent is $\alpha \approx 0.7$. Therefore this work lies in the opposite regime of $\alpha \ll \alpha^{*}$, where the long-range coupling terms are dominant over the magnetic field energy, and an analytical prediction can be obtained, as we will show below.

In the global coupling limit $(\alpha=0)$, the Hamiltonian

$$
H_{\alpha=0}=-B S_{N}^{z}+2 J_{0}\left(S_{N}^{x}\right)^{2}+N J_{0} / 2
$$

is called Lipkin-Meshkov-Glick model [59], which is integrable $[60,61]$ since there exist $N$ conserved quantities. For example, $\vec{S}_{n}^{2} \equiv S_{n}^{x 2}+S_{n}^{y 2}+S_{n}^{z 2}(n=2, \ldots, N)$ and the Hamiltonian (4) itself satisfy $\left[\vec{S}_{n}^{2}, H_{\alpha=0}\right]=0$, where $S_{n}^{\beta} \equiv$ $\sum_{i=1}^{n} \frac{1}{2} \sigma_{i}^{\beta}$ with $\beta=x, y, z$. In the special case of $\lambda \rightarrow \infty$ $(B \rightarrow 0)$, we can label each energy level by $\left|S_{1}, S_{2} \cdots S_{N-1}, S_{N}, S_{N}^{x}\right\rangle$ and group all the eigenstates into $N+1$ subspaces according to $S_{N}^{x}$. In each $S_{N}^{x}$ subspace, there are $\left(\begin{array}{c}N \\ N / 2+S_{N}^{x}\end{array}\right)$ degenerate levels. We define the notation $\left|\Phi_{(N / 2), S_{N}^{x}}^{\lambda=\infty}\right\rangle$ as the eigenstate with $S_{N}=N / 2$ and spin projection $S_{N}^{x}$ at $\lambda=\infty$.

For finite $\alpha>0$, since the interaction term in Hamiltonian (1) keeps the total spin projection $S_{N}^{x}$ unchanged, the eigenstates in different $S_{N}^{x}$ subspaces are decoupled. All the degenerate eigenstates in the same $S_{N}^{x}$ subspace couple each other resonantly and form new hybridized eigenstates $\left|\Phi_{n}\right\rangle$ appearing in Eq. (2). To estimate $\sigma_{A}$ in Eq. (3), we assume each many-body eigenstate $\left|\Phi_{n}\right\rangle$ to be a superposition of all the $\left(\begin{array}{c}N \\ N / 2+S_{N}^{x}\end{array}\right)$ levels in the $S_{N}^{x}$ subspace with probabilities fluctuating about their uniformly distributed value $\left(\begin{array}{c}N \\ N / 2+S_{N}^{x}\end{array}\right)^{-1}$. In the experiment, the prequenched spin state is $|\psi(0)\rangle=|\downarrow, \downarrow \cdots \downarrow\rangle_{z}$ which only couples the states with total spin $S_{N}=N / 2$. Since $\left|\Phi_{(N / 2), S_{N}^{X}}^{\lambda=\infty}\right\rangle$ is the only component with total spin $S_{N}=N / 2$ of the many-body $\left|\Phi_{n}\right\rangle$ in the $S_{N}^{x}$ subspace, we have

$$
\left|\left\langle\psi(0) \mid \Phi_{n}\right\rangle\right|^{2} \approx\left(\begin{array}{c}
N \\
N / 2+S_{N}^{x}
\end{array}\right)^{-1} P_{(N / 2), S_{N}^{x}}^{\lambda=\infty},
$$

with $\quad P_{(N / 2), S_{N}^{x}}^{\lambda=\infty} \equiv\left|\left\langle\psi(0) \mid \Phi_{(N / 2), S_{N}^{x}}^{\lambda=\infty}\right\rangle\right|^{2}$. Based on this assumption and the eigenstate thermalization hypothesis (ETH) $[8,9,56-58]$, we are able to obtain an approximate formula for Eq. (3) [42]

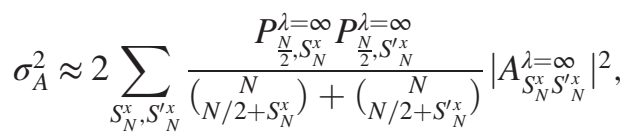

with the matrix element $A_{S_{N}^{x} S_{N}^{\prime x}}^{\lambda=\infty} \equiv\left\langle\Phi_{(N / 2), S_{N}^{x}}^{\lambda=\infty}|A| \Phi_{(N / 2), S_{N}^{\prime x}}^{\lambda=\infty}\right\rangle$. For large $N$, we have the asymptotic expression that
$\left(\begin{array}{c}N \\ N / 2+S_{N}^{x}\end{array}\right) \sim 2^{N} \sqrt{(2 / N \pi)} e^{-2\left(S_{N}^{x}\right)^{2} / N}$. The denominator of Eq. (6) indicates that $\sigma_{A} \propto 2^{-N / 2}$, predicting the size scaling exponent $\kappa=\ln \sqrt{2} \approx 0.35$.

Considering both $\lambda$ and $\alpha$ finite, the formula (6) holds as long as $\alpha \ll \alpha^{*}$ but the eigenstate $\left|\Phi_{(N / 2), S_{N}^{x}}^{\lambda}\right\rangle$ refers to the eigenlevel adiabatically connected to $\left|\Phi_{(N / 2), S_{N}^{x}}^{\lambda=\infty}\right\rangle$. In general, there is no simple closed form for the eigenstate $\left|\Phi_{(N / 2), S_{N}^{x}}^{\lambda}\right\rangle$ with a finite $\lambda$. However, Eq. (6) reduces the calculation of $\sigma_{A}$ to an $N \times N$ eigenvalue problem which can easily be solved on a computer [62]. As we will show further below, the analytical predictions compare well with the experiment (see Figs. 4(a) and 4(b) and Ref. [42]).

Experimental results.-To perform this experiment, we use a trapped-ion quantum simulator [43] where each effective spin-1/2 particle is encoded in the hyperfine ground state of one ${ }^{171} \mathrm{Yb}^{+}$ion with $|\uparrow\rangle \equiv$ ${ }^{2} \mathrm{~S}_{1 / 2}\left|F=1, m_{F}=0\right\rangle$ and $|\downarrow\rangle \equiv{ }^{2} \mathrm{~S}_{1 / 2}\left|F=0, m_{F}=0\right\rangle$. The Hamiltonian of Eq. (1) is realized by global spindependent optical dipole forces from laser beams, which modulate the Coulomb interaction to create an effective Ising coupling between spins [63]. The field term is implemented by asymmetrically detuning the two laser beat notes generating the optical dipole forces [42].

The magnetization fluctuations $\sigma_{A}$ are characterized by measuring the standard deviation of the average magnetization of the sum of all ions in the chain, i.e., $\langle A\rangle=N^{-1} \sum_{j}\left\langle\sigma_{j}^{z}\right\rangle$. This is measured with $B$ fields ranging from $\pm 2 \pi \times 0.5 \mathrm{kHz}$ to $2 \pi \times 2.0 \mathrm{kHz}$. The two plots in Fig. 2 show the magnetization data measured as a function of time with a four-ion chain and $B= \pm 2 \pi \times 0.5 \mathrm{kHz}$. Although the decoherence time in our trapped-ion simulator is long enough to consider $J_{0}$ and $B$ unchanged within a single time evolution up to $t=2 \mathrm{~ms}$, the values of $J_{0}$ and $B$ may vary between different time evolutions. We assume the coupling strength and magnetic field in the experiments to be independent and normally distributed. Then, the averaged observable $A$ at a fixed time $t$ also needs to be averaged over the experimental values of $J_{0}$ and $B$, resulting in

$$
\langle A(t)\rangle=\langle\langle\psi(t)|A| \psi(t)\rangle\rangle_{J_{0}, B} .
$$

In Fig. 2, the red curves are the theory fits by setting $\sigma_{J_{0}}$ and $\sigma_{B}$ both to approximately $2 \pi \times 0.1 \mathrm{kHz}$. To fit the experimental data, we use the gradient descent method to search for the optimal values of $\sigma_{J_{0}}$ and $\sigma_{B}$, which happen to be roughly equal. Therefore, we set $\sigma_{J_{0}}$ and $\sigma_{B}$ to be the same values for simplicity.

In general, with a positive $B$ field, we observe more significant oscillations than when using a negative $B$ field. This can be understood by analyzing the overlap between the prequench state and the postquench energy eigenstates (obtained for the postquench $J_{0}$ and $B$ values). For the 

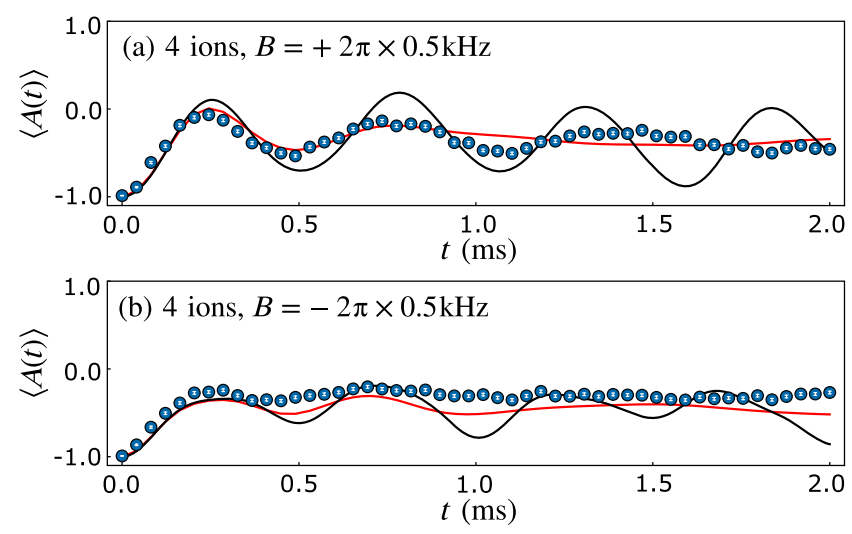

FIG. 2. Time evolution of average magnetization, $\langle A\rangle=N^{-1} \sum_{j}\left\langle\sigma_{j}^{z}\right\rangle$, over $N=4$ ions out to $2 \mathrm{~ms}$ for $B=+2 \pi \times$ $0.5 \mathrm{kHz}$ (a) and $B=-2 \pi \times 0.5 \mathrm{kHz}$ (b). Each data point is the average of 4000 experiments, reported with the respective statistical error (white bars). For both plots: blue are data points, black and red are theoretical results with $\left(\sigma_{J_{0}}, \sigma_{B}\right)=0$ and $\left(\sigma_{J_{0}}, \sigma_{B}\right)=2 \pi \times(0.1,0.1) \mathrm{kHz}, \quad$ respectively. Parameters: $J_{0}=2 \pi \times 0.50 \mathrm{kHz}, \alpha=0.71$.

system parameters given in Fig. 2, the structure of the postquench spectrum is such that at high energies there is a nonvanishing energy gap in the thermodynamic limit. Conversely, in the low energy sector of the spectrum the level spacing decreases with system size and the gap vanishes in the thermodynamic limit. For the positive $B$ field, the prequench state is the superposition of several of the highest excited states of the spectrum and the energy gap leads to more persistent oscillations. For the negative $B$ field, the prequench state is very close to the ground state of the spectrum [64], suppressing the oscillations [42].

We plot the standard deviation of the average magnetization $\sigma_{A}$ as a function of $\lambda=2 J_{0} / B$ for fixed $N$ in Fig. 3 . The data for $N=3$ to $N=6$ agree with the theoretical prediction. The $N=7$ data largely agrees with theory excluding the two outlying points at negative $\lambda$ values. For $N=8$, the data points tend to gather around the 0.07 level indicating that the measurement noise in this case obscures the measured fluctuations. In these plots, the values near $\lambda=0$ were not taken because when $B \gg J_{0}$ the ions are predominantly acting paramagnetically. In this regime, fluctuations are expected to be very small and well below the noise floor of this experiment. The shape of the data is asymmetric with a pronounced slope at $2 J_{0} / B=1 / 2$. This point marks the ferromagnetic (FM) to paramagnetic (PM) phase transition of the ion chain. The fluctuations are enhanced here as this is an unstable point for the system. In contrast, the antiferromagnetic (AFM) to PM transition [31] for $\lambda<0$ is not as pronounced.

System size scaling.-The temporal fluctuation variance $\sigma_{A}^{2}$ given by Eq. (3) is obtained by averaging over an infinite time window $J_{0} t \in[0,+\infty]$. However, in the experiment, we can only average over a finite time window up to $t \sim 2.0 \mathrm{~ms}$ (i.e., 3 or 4 oscillations depending on the value of $\lambda$ ), as the long-time fluctuations are suppressed by the noise in the parameters $J_{0}$ and $B$. In Figs. 4(a)-4(c), we compare the analytical results given by Eq. (6) to the numerical results with different averaging time windows. The short-time-window averaging only makes sense for small system size as larger system sizes result in smaller
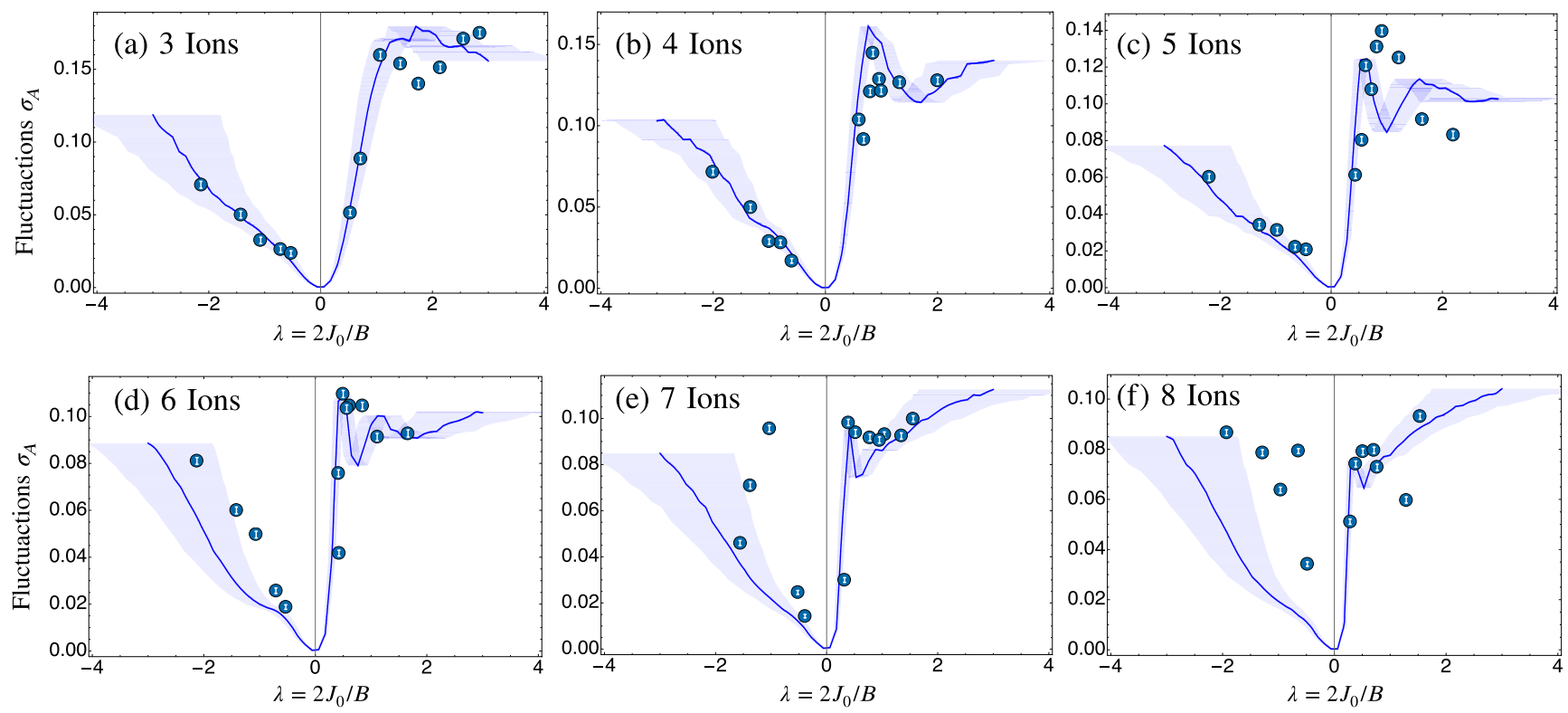

FIG. 3. Temporal fluctuation $\sigma_{A}$ as a function of $\lambda=2 J_{0} / B$ for $N=3-8$ ions from experimental data (blue dots with white error bars) and from numerical simulations (blue curves) with parameters: $\left(\sigma_{J_{0}}, \sigma_{B}\right)=2 \pi \times(0.12,0.12) \mathrm{kHz}$ for $N=3,2 \pi \times(0.11,0.11) \mathrm{kHz}$ for $N=5$ and $2 \pi \times(0.10,0.10) \mathrm{kHz}$ for $N=4,6-8$. The blue shade associated with each numerical curve takes account of the experimental uncertainty of $\lambda$. Experimental parameters: see Table I in the Supplemental Material [42]. 

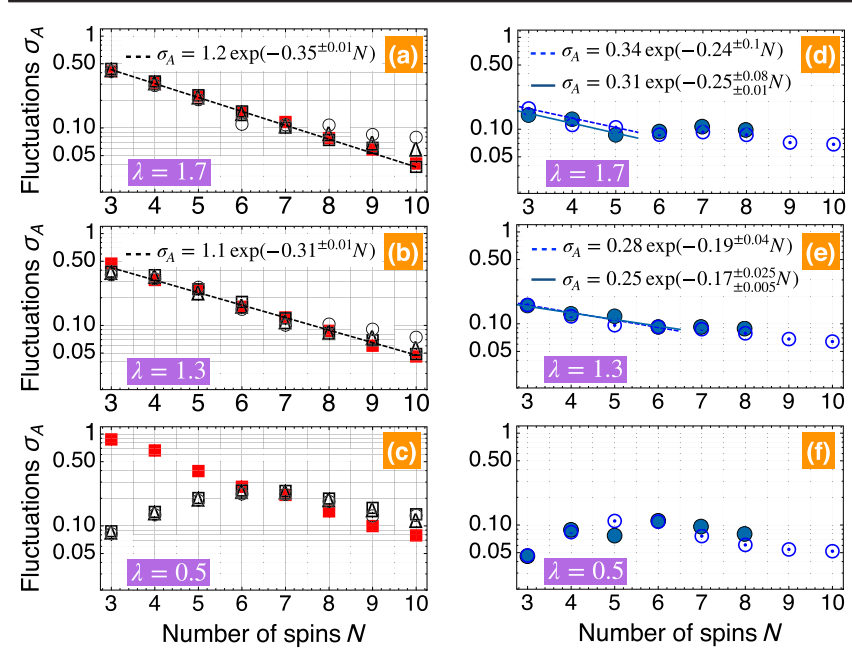

FIG. 4. Logplot of temporal fluctuation $\sigma_{A}$ versus number of spins $N$ for different $\lambda$ values. (a)-(c) Analytical results (red squares) vs numerical results with different time windows $J t \in$ $[0,2 \pi]$ (circles), $J t \in[0,5 \pi]$ (triangles), and $J t \in[0, \infty]$ (empty squares) for $\alpha=0.7$. (d)-(f) Experimental results (blue dots) vs numerical results (circled dots) taken from Fig. 3. The dashed and solid lines are the fits.

level splittings and makes the period of temporal fluctuations longer. The fits to the infinite-time-window averaging show that the system size scaling exponent is $\kappa \approx 0.31$ $(\kappa \approx 0.35)$ for $\lambda=1.3(\lambda=1.7)$, which is close to the theoretical prediction $\kappa=\ln \sqrt{2}$ for $N \gg 1$ [42].

In Figs. 4(d)-4(f), we compare the experimental data with the numerical results taken from Fig. 3 for different $\lambda$ values. The fits to the experimental data and numerical results for $N=3-5(\lambda=1.7)$ or $N=3-6(\lambda=1.3)$ show good agreement. For $\lambda=0.5$, our analytical expression breaks down as the system is in the crossover regime, but the experimental data still confirm the numerical simulations as shown in Fig. 4(f). For $\lambda=1.7$, the fit to the experimental data gives the system size scaling exponent $\kappa=0.25_{ \pm 0.01}^{ \pm 0.08}$, where the superscript is the uncertainty from the least square fitting and the subscript is the uncertainty from the statistical errors in the experiments [42]. We finally note that an exponential fit of data generated from single-particle dephasing $\left(\sigma_{A} \propto 1 / \sqrt{N}\right)$ [39] for $N=3-5$ would lead to an exponent $\kappa \sim 0.13$, which is expected to be even further suppressed by the noise in $J_{0}$ an $B$. A detailed statistical analysis is presented in the Supplemental Material [42].

Summary.-Using a trapped-ion quantum simulator, we have presented the first experimental observation of persistent temporal fluctuations after a quantum quench with a long-range interacting transverse-field Ising model. We characterized how the fluctuations in the average magnetization of the spin chain depend on the transverse field and the spin-spin interactions. Numerical simulations compared with experiment show that, as a function of system size $N$, the exponential suppression of temporal fluctuations matches well with the theoretical prediction.

This work is supported by the National Science Foundation (NSF) Practical Fully-Connected Quantum Computer (PFCQC) STAQ program, the Air Force Office of Scientific Research (AFOSR) Multidisciplinary University Research Initiatives (MURI) on Quantum Measurement and Verification $(\mathrm{QM} \& \mathrm{~V})$ and Dissipatively Stabilized Qubits and Materials, the Defense Advanced Research Projects Agency (DARPA) program on Driven and Nonequilibrium Quantum Systems (DRINQS), the Department of Energy (DOE) BES Materials and Chemical Sciences for Quantum Information Science program (Award No. DE-FOA0001909) for optical system upgrades, and the DOE HEP QuantISED Program (Award No. DE-FOA0001893) for cryogenic vacuum chamber maintenance.

\footnotetext{
*These authors contributed equally to this work.

Corresponding author.

lingzhen.guo@mpl.mpg.de

Corresponding author. monroe@umd.edu
}

[1] T. Kinoshita, T. Wenger, and D. Weiss, A quantum Newton's cradle, Nature (London) 440, 900 (2006).

[2] A. Polkovnikov, K. Sengupta, A. Silva, and M. Vengalattore, Colloquium: Nonequilibrium dynamics of closed interacting quantum systems, Rev. Mod. Phys. 83, 863 (2011).

[3] C. Gogolin and J. Eisert, Equilibration, thermalisation, and the emergence of statistical mechanics in closed quantum systems, Rep. Prog. Phys. 79, 056001 (2016).

[4] H. Bernien, S. Schwartz, A. Keesling et al., Probing manybody dynamics on a 51-atom quantum simulator, Nature (London) 551, 579 (2017).

[5] M. Rigol, V. Dunjko, and M. Olshanii, Thermalization and its mechanism for generic isolated quantum systems, Nature (London) 452, 854 (2008).

[6] J. Eisert, M. Friesdorf, and C. Gogolin, Quantum manybody systems out of equilibrium, Nat. Phys. 11, 124 (2015).

[7] C. Neuenhahn and F. Marquardt, Thermalization of interacting fermions and delocalization in Fock space, Phys. Rev. E 85, 060101(R) (2012).

[8] J. M. Deutsch, Quantum statistical mechanics in a closed system, Phys. Rev. A 43, 2046 (1991).

[9] M. Srednicki, Chaos and quantum thermalization, Phys. Rev. E 50, 888 (1994).

[10] C. Kollath, A. M. Läuchli, and E. Altman, Quench Dynamics and Nonequilibrium Phase Diagram of the Bose-Hubbard Model, Phys. Rev. Lett. 98, 180601 (2007).

[11] M. Cramer, C. M. Dawson, J. Eisert, and T. J. Osborne, Exact Relaxation in a Class of Nonequilibrium Quantum Lattice Systems, Phys. Rev. Lett. 100, 030602 (2008).

[12] S. Trotzky, Y. Chen, A. Flesch, I. P. McCulloch, U. Schollwöck, J. Eisert, and I. Bloch, Probing the relaxation 
towards equilibrium in an isolated strongly correlated onedimensional Bose gas, Nat. Phys. 8, 325 (2012).

[13] M. Gring, M. Kuhnert, T. Langen, T. Kitagawa, B. Rauer, M. Schreitl, I. Mazets, D. A. Smith, E. Demler, and J. Schmiedmayer, Relaxation and prethermalization in an isolated quantum system, Science 337, 1318 (2012).

[14] D. A. Smith, M. Gring, T. Langen, M. Kuhnert, B. Rauer, R. Geiger, T. Kitagawa, I. Mazets, E. Demler, and J. Schmiedmayer, Prethermalization revealed by the relaxation dynamics of full distribution functions, New J. Phys. 15, 075011 (2013).

[15] T. Langen, R. Geiger, M. Kuhnert, B. Rauer, and J. Schmiedmayer, Local emergence of thermal correlations in an isolated quantum many-body system, Nat. Phys. 9, 640 (2013).

[16] T. Langen, S. Erne, R. Geiger, B. Rauer, T. Schweigler, M. Kuhnert, W. Rohringer, I. E. Mazets, T. Gasenzer, and J. Schmiedmayer, Experimental observation of a generalized Gibbs ensemble, Science 348, 207 (2015).

[17] A. M. Kaufman, M.E. Tai, A. Lukin, M. Rispoli, R. Schittko, P. M. Preiss, and M. Greiner, Quantum thermalization through entanglement in an isolated many-body system, Science 353, 794 (2016).

[18] G. Clos, D. Porras, U. Warring, and T. Schaetz, Time-Resolved Observation of Thermalization in an Isolated Quantum System, Phys. Rev. Lett. 117, 170401 (2016).

[19] B. Neyenhuis, J. Zhang, P. W. Hess, J. Smith, A. C. Lee, P. Richerme, Z.-X. Gong, A. V. Gorshkov, and C. Monroe, Observation of prethermalization in long-range interacting spin chains, Sci. Adv. 3, e1700672 (2017).

[20] M. Schreiber, S. S. Hodgman, P. Bordia, H. P. Luschen, M. H. Fischer, R. Vosk, E. Altman, U. Schneider, and I. Bloch, Observation of many-body localization of interacting fermions in a quasirandom optical lattice, Science 349, 842 (2015).

[21] J. Smith, A. Lee, P. Richerme, B. Neyenhuis, P. W. Hess, P. Hauke, M. Heyl, D. A. Huse, and C. Monroe, Many-body localization in a quantum simulator with programmable random disorder, Nat. Phys. 12, 907 (2016).

[22] J.-y. Choi, S. Hild, J. Zeiher, P. Schauss, A. Rubio-Abadal, T. Yefsah, V. Khemani, D. A. Huse, I. Bloch, and C. Gross, Exploring the many-body localization transition in two dimensions, Science 352, 1547 (2016).

[23] This crossover may be related to the phenomenon of dynamical phase transition [24-30]. But it is not the focus of the present Letter, so we leave its study to future investigations. The crossover value of $\alpha^{*}=\ln (2|\lambda|) / \ln 2$ can be obtained by comparing the magnetic field energy and the NNN coupling term, i.e., $\left|2^{-\alpha^{*}+2} J_{0}\right|=|-B|$. Note that this crossover is different from the well-known paramagnetic to antiferromagnetic phase transition discussed in Ref. [31].

[24] M. Heyl, A. Polkovnikov, and S. Kehrein, Dynamical Quantum Phase Transitions in the Transverse-Field Ising Model, Phys. Rev. Lett. 110, 135704 (2013).

[25] M. Heyl, Dynamical Quantum Phase Transitions in Systems with Broken-Symmetry Phases, Phys. Rev. Lett. 113, 205701 (2014).

[26] J. Zhang, G. Pagano, P. W. Hess, A. Kyprianidis, P. Becker, H. Kaplan, A. V. Gorshkov, Z.-X. Gong, and C. Monroe,
Observation of a many-body dynamical phase transition with a 53-qubit quantum simulator, Nature (London) 551, 601 (2017).

[27] J. C. Halimeh, V. Zauner-Stauber, I. P. McCulloch, I. de Vega, U. Schollwöck, and M. Kastner, Prethermalization and persistent order in the absence of a thermal phase transition, Phys. Rev. B 95, 024302 (2017).

[28] J. C. Halimeh and V. Zauner-Stauber, Dynamical phase diagram of quantum spin chains with long-range interactions, Phys. Rev. B 96, 134427 (2017).

[29] V. Zauner-Stauber and J.C. Halimeh, Probing the anomalous dynamical phase in long-range quantum spin chains through Fisher-zero lines, Phys. Rev. E 96, 062118 (2017).

[30] B. Żunkoviê, M. Heyl, M. Knap, and A. Silva, Dynamical Quantum Phase Transitions in Spin Chains with LongRange Interactions: Merging Different Concepts of Nonequilibrium Criticality, Phys. Rev. Lett. 120, 130601 (2018).

[31] T. Koffel, M. Lewenstein, and L. Tagliacozzo, Entanglement Entropy for the Long-Range Ising Chain in a Transverse Field, Phys. Rev. Lett. 109, 267203 (2012).

[32] L. C. Venuti and P. Zanardi, Gaussian equilibration, Phys. Rev. E 87, 012106 (2013).

[33] A. C. Cassidy, C. W. Clark, and M. Rigol, Generalized Thermalization in an Integrable Lattice System, Phys. Rev. Lett. 106, 140405 (2011).

[34] C. Gramsch and M. Rigol, Quenches in a quasidisordered integrable lattice system: Dynamics and statistical description of observables after relaxation, Phys. Rev. A 86, 053615 (2012).

[35] P. Reimann, Foundation of Statistical Mechanics under Experimentally Realistic Conditions, Phys. Rev. Lett. 101, 190403 (2008).

[36] A. J. Short and T. C. Farrelly, Quantum equilibration in finite time, New J. Phys. 14, 013063 (2012).

[37] L. Campos Venuti and P. Zanardi, Universal time fluctuations in near-critical out-of-equilibrium quantum dynamics, Phys. Rev. E 89, 022101 (2014).

[38] E. J. Torres-Herrera, D. Kollmar, and L. F. Santos, Relaxation and thermalization of isolated many-body quantum systems, Phys. Scr. T165, 014018 (2015).

[39] T. Kiendl and F. Marquardt, Many-Particle Dephasing after a Quench, Phys. Rev. Lett. 118, 130601 (2017).

[40] P. R. Zangara, A. D. Dente, E. J. Torres-Herrera, H. M. Pastawski, A. Iucci, and L.F. Santos, Time fluctuations in isolated quantum systems of interacting particles, Phys. Rev. E 88, 032913 (2013).

[41] In Ref. [39], the authors used the name "many-particle dephasing" since their model can be reduced into weakly interacting quasiparticles. But for the model considered in our work, there is no clear quasiparticle picture. Thus, we rephrase the name as "many-body dephasing" here.

[42] See the Supplemental Material at http://link.aps.org/ supplemental/10.1103/PhysRevLett.125.120605 for details, which includes Refs. [8,9,39,43-58].

[43] G. Pagano et al., Cryogenic trapped-ion system for large scale quantum simulation, Quantum Sci. Technol. 4, 014004 (2019).

[44] E.P. Wigner, Characteristic vectors of bordered matrices with infinite dimensions, Ann. Math. 62, 548 (1955); 
Characteristics vectors of bordered matrices with infinite dimensions II, Ann. Math. 65, 203 (1957); On the distribution of the roots of certain symmetric matrices, Ann. Math. 67, 325 (1958).

[45] F. J. Dyson, Statistical theory of the energy levels of complex systems. I, J. Math. Phys. (N.Y.) 3, 140 (1962).

[46] M. L. Mehta, Random Matrices (Elsevier/Academic Press, Amsterdam, 2004).

[47] L. F. Santos and M. Rigol, Onset of quantum chaos in onedimensional bosonic and fermionic systems and its relation to thermalization, Phys. Rev. E 81, 036206 (2010).

[48] V. K. B. Kota, Embedded Random Matrix Ensembles in Quantum Physics (Springer, Cham, 2014), p. 31, ISBN: 978-3-319-04567-2.

[49] M. V. Berry, Regular and irregular semiclassical wavefunctions, J. Phys. A 10, 2083 (1977).

[50] K. Molmer and K. Sorensen, Multiparticle Entanglement of Hot Trapped Ions, Phys. Rev. Lett. 82, 1835 (1999).

[51] K. Kim, M. S. Chang, R. Islam, S. Korenblit, L. M. Duan, and C. Monroe, Entanglement and Tunable Spin-Spin Couplings between Trapped Ions Using Multiple Transverse Modes, Phys. Rev. Lett. 103, 120502 (2009).

[52] A. C. Lee, Engineering a quantum many-body Hamiltonian with trapped ions, Aaron Lee Thesis, 2016.

[53] S. Olmschenk, K. C. Younge, D. L. Moehring, D. Matsukevich, P. Maunz, and C. Monroe, Manipulation and detection of a trapped $\mathrm{Yb}+$ hyperfine qubit, Phys. Rev. A 76, 052314 (2007).

[54] C.-C. Joseph Wang and J. K. Freericks, Intrinsic phonon effects on analog quantum simulators with ultracold trapped ions, Phys. Rev. A 86, 032329 (2012).
[55] Y. Wu, thesis, University of Michigan, 2019.

[56] M. Srednicki, The approach to thermal equilibrium in quantized chaotic systems, J. Phys. A 32, 1163 (1999).

[57] W. Beugeling, R. Moessner, and M. Haque, Off-diagonal matrix elements of local operators in many-body quantum systems, Phys. Rev. E 91, 012144 (2015).

[58] T. Mori, T. N. Ikeda, E. Kaminishi, and M. Ueda, Thermalization and prethermalization in isolated quantum systems: A theoretical overview, J. Phys. B 51, 112001 (2018).

[59] H. Lipkin, N. Meshkov, and A. Glick, Validity of many-body approximation methods for a solvable model: (I). Exact solutions and perturbation theory, Nucl. Phys. 62, 188 (1965).

[60] H. Morita, H. Ohnishi, J. da Providência, and S. Nishiyama, Exact solutions for the LMG model Hamiltonian based on the Bethe ansatz, Nucl. Phys. B737, 337 (2006).

[61] W. Miller, Jr., S. Post, and P. Winternitz, Classical and quantum superintegrability with applications, J. Phys. A 46, 423001 (2013).

[62] J. A. Alexander, P. Reinhard, and E. Suraud, Simple Models of Many-Fermion Systems (Spring-Verlag, Berlin, Heidelberg, 2010), p. 171.

[63] C. Monroe et al., Programmable quantum simulations of spin systems with trapped ions, arXiv:1912.07845.

[64] P. Jurcevic, H. Shen, P. Hauke, C. Maier, T. Brydges, C. Hempel, B. P. Lanyon, M. Heyl, R. Blatt, and C. F. Roos, Direct Observation of Dynamical Quantum Phase Transitions in an Interacting Many-Body System, Phys. Rev. Lett. 119, 080501 (2017). 